\section{P4.69 HEALTH CARE SEEKING BEHAVIOUR FOR SYMPTOMS OF STIS/HIV AMONG THE TRANSGENDER IN MUMBAI, INDIA}

Mithlesh Chourase. International Institute for Population Sciences (IIPS), Mumbai, Mumbai, India

10.1136/sextrans-2017-053264.565

Introduction Health of the transgender is as important as any other population sub-groups. However, little is known about the issues of health problems and health seeking behaviour of transgender in India. One of the major risk factors for acquisition of HIV and other STIs for MTF transgender persons is Commercial sex workers (CSW). As with HIV infection in this population, it is difficult to ascertain the exact prevalence of CSW in MTF transgender persons. This study is an attempt to assess the health care seeking behaviour of women reporting STIs/HIV symptoms in mumbai, India and to examine the association of background sociodemographic characteristics with Health care seeking behaviour.

Methodology The study used the primary survey data conducted in Mumbai city among the transgender community with a total sample of 120 . Both qualitative and quantitative data was collected on demographic and socio-economic characteristic, sexual health problems and health seeking behaviour among transgender.

Results Among the transgender, the prevalence of sexual health is very high. In this community $28 \%$ and $21.7 \%$ of the transgender are suffered from STI and HIV respectively. Among those who suffered from any sexual health problems, $7 \%$ of the transgender do not take any treatment. Those who seek treatment for sexual health problems most of them go to government medical institution because of low treatment cost compared to a private institution. On the other hand, those who don't seek treatment revealed that they don't prefer to take treatment because of their personal problem or due to shame, high medicine cost and few believe in yoga.

Conclusion Though the majority of transgender heard about STI, the correct knowledge of sexual health transmission is lacking among the transgender. There is a need for increasing awareness regarding STI/HIV and their sequelae. Targeted health education programmes should be necessary to improve health care seeking among transgender.

\section{P4.70 AVERTING AN IMPENDING HIV EPIDEMIC: MASSIVE INTERVENTIONS NEEDED FOR INJECTING DRUG USERS (IDUS) IN BANGLADESH}

Mohammed Abul Kalam. Siam Health Care, Dhaka, Bangladesh

10.1136/sextrans-2017-053264.566

Introduction Far from being an isolated population, injectors are intimately connected with others. They are linked with the rest of society - they have regular sex partners, they buy sex from women, as well as other men, they sell blood, and they also move between cities and inject. As long as HIV prevention through safe behaviour is not norm, the rest society is at risk from this virus. This study aims to collect information on IDUs and their health seeking behaviour in Bangladesh.

Methods A cross-sectional study was conducted among 120 IDUs attending a drug rehabilitation centre in Rajshahi, Bangladesh. Data were collected on socio-demographics, drug use, health seeking behaviour, knowledge of injecting drugs, and sexual behaviour.

Results The mean \pm SD and median (IQR) age of the participants were $32.5 \pm 21.3$ and 33 (27-38) years, respectively, with only $9.2 \%$ females. Injection buprenorphine was the drug of choice for $40 \%$ of participants, and $58 \%$ of the participants first started drug use with smoking cannabis. $73.3 \%$ of participants shared needles sometimes and $57.5 \%$ were willing to use the needle exchange programs. $60 \%$ of the participants had no knowledge about the diseases spread by injection. Condom use during the last intercourse with regular partners was $11.7 \%$ and with any partners $15.8 \%$.

Conclusion IDUs in Bangladesh are a high-risk group for HIV/ AIDS due to lack of knowledge and risky behaviour. Bangladesh must immediately make it high priority to scale up interventions massively and stop further spread of HIV among drug injectors and other vulnerable people. And Bangladesh must act rapidly and decisively to avert the impending HIV epidemic or it will be too late.

\section{P4.71 THE POWER OF PEOPLE LIVING WITH HIV (PLHIV) IN STIGMA REDUCTION}

Mohammed Ahmed Taha Elzaki. UNFPA, Khartoum, Sudan

10.1136/sextrans-2017-053264.567

This paper briefly reflects the fact that People living with HIV/ AIDS (PLHIV) can be potential resources for HIV-related stigma reduction, the paper shares the experience of Sudanese People living With HIV/AIDS Care Association (SPCA) on reducing selfsigma among the PLHIV themselves and on the other hand reducing the stigma among health service providers and the general population. In the near past, Stigma was the major challenge constraining HIV intervention efforts in Sudan where the social context is one of the generating factor for the stigma and discrimination, the type of stigma in Sudan was the most furious one which influence PLHIV and their families, it was a complex model of stigma that deny PLHIV to even access care and treatment. The efforts made to empower PLHIV were a breakthrough towards better PLHIV-friendly environment free of stigma and discrimination. This paper focuses on the impact of the PLHIV efforts in the stigma reduction, which has contributed to put PLHIV and AIDS issues in the primary agenda of the government commitment and helped to advocate for supporting the overall intervention of AIDS in the country. The paper also shows how far the role of other sectors contributed to empower PLHIV and helped the national AIDS Programme to apply mature strategies on stigma reduction and comprehensive programming for HIV response.

\section{P4.72 STIGMA AND DISCRIMINATION EXPERIENCED BY YOUNG MEN WHO HAVE SEX WITH MEN IN LARGE CITIES OF MYANMAR}

Myo Myo Mon, Kyaw Min Htut, Wai Wai Myint. Ministry of Health and Sports, Yangon, Myanmar Burma

\subsection{6/sextrans-2017-053264.568}

Introduction Young men who have sex with men (YMSM) are experiencing stigma and discrimination ( $S$ and $D)$ resulting in social exclusion with adverse consequences. Therefore, a study 
was conducted among YMSM in two large cities of Myanmar in 2014 with the objective of determining the pattern of $S$ and D experienced by YMSM.

Methods A cross-sectional study was conducted by using quantitative and qualitative research methods. Respondent driven sampling was applied to recruit YMSM. Face-to-face interviews were done by using a structured questionnaire and in-depth interviews were also done with YMSM.

Results A total of 400 YMSM (200 each in Yangon and Monywa) included in the study. Their age ranged from 18-26 years with the median age of 22 . Over $42 \%$ self-identified as Apwint (feminine). Most YMSM (55.8\% and 69.6\%) reported having a regular job, but a higher percentage in Monywa reported an estimated monthly income of $>100,000$ Kyats compared to YMSM in Yangon $(\mathrm{p}<0.01)$. Around half $(48.2 \%$ and $51.5 \%)$ reported ever experiencing discrimination because of their same sex attraction. Among those who experienced discrimination, 60\% in Yangon and 53\% in Monywa reported experiencing discrimination exhibited as insults/verbal abuse and $(34 \%$ and $37 \%)$ reported discrimination exhibited as physical abuse or beating. Seventy percent of YMSM reported experiencing discrimination most often in public places. The largest percentages of YMSM who reported ever experiencing discrimination, were among Apwint (64\% and 50\%). "Exclusion from social occasion" and "beating" were indicated as the worst type of discrimination by the many YMSM in Yangon $(40 \%$ vs. $15 \%, \mathrm{p}<0.01)$ and Monywa $(47 \%$ vs. $27 \%$, $\mathrm{p}>0.05)$. Some YMSM stated that they are experiencing $S$ and D even among the family members and sometimes at health care settings resulting in delayed seeking care. They thought that people would not discriminate them if they become famous and could earn much money.

Conclusion S and D still existed towards YMSM especially in public places. Community awareness raising programs should be strengthened to reduce S and D towards YMSM.

\section{P4.73 ATTITUDES AND PRACTICES OF NURSES UPON THE DELIVERY OF HEALTH CARE TO COMMERCIAL SEX WORKERS (CSW) IN THE WESTERN PROVINCE, SRI LANKA}

${ }^{1} \mathrm{~N}$ Jayasinghe, ${ }^{2} \mathrm{~S}$ Tissera, ${ }^{3} \mathrm{~N}$ Silva,. ${ }^{1}$ Deputy Head - Nursing Faculty, International Institute of Health Sciences, Sri Lanka; ${ }^{23}$ rd year student, Nursing Faculty, International Institute of Health Sciences, Sri Lanka; ${ }^{3}$ Head of Academic Affairs, International Institute of Health Sciences, Sri Lanka

\subsection{6/sextrans-2017-053264.569}

Introduction The traditional socio-cultural norms of countries like Sri Lanka pose challenges to health care access to those engaged in tabooed and illegal professions like the commercial sex trade. CSWs accessing health services in similar countries have been proven to be of a low socio-economic background. Therefore it is important to prompt a behavioural change by placing strong policies in place to address the psychosocial issues of both the patient and the carer. Certain STI's like HIV, being immuno-compromising in nature, increase the susceptibility of many other illnesses, to which these CSWs should seek treatment promptly. In order to make policy level decisions to tailor the training of health care workers to improve acceptance of CSWs at their settings, it is first important to assess the current gaps in delivering holistic care to CSWs. The goal was to understand attitudes and perceptions of nurses (in multi-sectors) when attending to sexual and general health needs and delivering holistic health care to CSWs in the Western Province of Sri Lanka.

Methods A qualitative research which consisted of 3 focus group discussions were carried out on a total of 23 nurses from the government and private sectors where each group consisted of 7-8 participants with 2 moderators per group. Scenarios and questions were posed to evaluate the perceptions of nurses towards CSWs when delivering STI related and general health care.

Results Thematic analysis revealed three strong themes. They were, 'No difference in the level of care delivered', 'Social marginalisation based on assumptions' and 'Labelling and stereotyping of CSWs'. No difference was made in the level of care provided for CSWs compared to non-CSWs, or for STIs compared to other disease conditions. Though the level of care delivered is the same, the manner in which CSWs are 'made comfortable' in terms of 'holistic care' within the hospitals seemed less compared to other patients. Labelling and stereotyping were common practices, where the nurses did believe that their attitudes and behaviour could have a negative affect on health promotion and health seeking behaviour.

Conclusion It is better to invest on training nurses so that the CSWs are made to feel more accepted when accessing STI related and general health care needs.

\section{P4.74 ABSTRACT WITHDRAWN}

\section{P4.75 EXPLORING THE RELATIONSHIP BETWEEN SOCIAL FACTORS AND TREATMENT ADHERENCE AMONG CLINICALLY STABLE ART PATIENTS: STRUCTURAL EQUATION MODELLING TECHNIQUE}

${ }^{1}$ Ndumiso Tshuma, ${ }^{2}$ Peter Nyasulu. 'Community AIDS Response, Johannesburg, South African Republic; ${ }^{2}$ Wits University, Johannesburg, South African Republic

\subsection{6/sextrans-2017-053264.570}

Introduction Socio-cultural factors such as fear of disclosure of HIV status, lack of social support and fear of stigma from family are some of the aspects that prevent ART adherence amongst patients. Moreover, home stability and family support have been linked to better ART adherence. One study has revealed that males who had long-term housing, living with a partner and belonged to an HIV support group had better antiretroviral adherence. The study sought to establish the relationship between social factors and treatment adherence among adherence clubs members.

Methods A cross-sectional survey was conducted among 830 Adherence Club patients in Ekurhuleni, Gauteng. A closedended questionnaire was administered and the internal consistency of the scale was assessed by Cronbach's alpha coefficient. The proposed model was tested using structural equation modelling (AMOS software: ADC, Chicago, IL, USA).

Results Adherence clubs were found to improve treatment adherence among patients. Patients who were in adherence clubs are also receiving more support from their family members or the people they stay with. Perceptions and experiences of stigma and discrimination were found to be generally low among patients attending adherence clubs. These low levels of stigma and discrimination lead to an improvement in the levels of treatment adherence among the club members. 